\title{
The Canadian LMC Diabetes Registry: A Profile of the Demographics, Management, and Outcomes of Individuals with Type 1 Diabetes
}

\author{
Ronnie Aronson, MD, Ruth E. Brown, PhD, Alexander Abitbol, MD, Ronald Goldenberg, MD, \\ Zeina Yared, MD, Buki Ajala, MBBS, and Jean-Francois Yale, MD
}

\begin{abstract}
Objective: Clinical guidelines now define the standard of diabetes care, but few health care jurisdictions systematically assess their practicality and impact. The Canadian LMC Diabetes Registry includes the electronic health records of $>50$ endocrinologists in three provinces and provides quarterly real-time outcome reports to each endocrinologist. This retrospective cohort study aimed to characterize the demographics, treatment regimens, and outcomes of the type 1 diabetes (T1D) patient population in the registry.

Research Design and Methods: Adults were included if they had a clinical diagnosis of T1D, had seen an LMC endocrinologist between July 1, 2015 and June 30, 2018, and had follow-up $>6$ months. This study is registered on clinicaltrials.gov (NCT04162067).

Results: The resulting cohort included 3600 individuals with mean age of $43.9 \pm 15.3$ years and duration of diabetes of $21.5 \pm 13.9$ years. Mean hemoglobin A1C (HbA1c) was $8.1 \% \pm 1.5 \%$ and only $22.5 \%$ had achieved $\mathrm{HbA} 1 \mathrm{c} \leq 7.0 \%$. In each measure, individuals in younger cohorts showed poorer glycemic control than older cohorts. Within each age cohort, insulin pump users showed a lower mean HbAlc than those using multiple daily injections, especially in cohorts who were also not using a continuous glucose monitor. Overall, $63.1 \%$ reported at least weekly hypoglycemia, whereas $3.6 \%$ reported severe hypoglycemia $\geq 1$ per year.

Conclusions: Despite receiving care in an advanced well-resourced environment, within a public health care system, from specialists armed with regular patient outcomes feedback, most individuals with T1D are unable to achieve the goals recommended by clinical practice guidelines.
\end{abstract}

Keywords: Type 1 diabetes, Insulin therapy, Glycemic control, Continuous glucose monitoring.

\section{Introduction}

D IABETES REPRESENTS A growing burden on global health care systems and is arguably unique as a chronic disease in that accessible current health outcome measures (e.g., glycemia, blood pressure, and lipids) directly predict the resulting health burden. As such, most health care authorities have developed or adopted comprehensive care guidelines to optimize these outcomes. However, few have taken the important step of maintaining a systematic awareness of those outcomes to reconcile the practicality and the impact of well-intended practice guidelines.

In type 1 diabetes (T1D), several large-scale population registries have emerged, including the Swedish National Diabetes Register (NDR), ${ }^{1}$ the Diabetes-PatienenVerlaufsdokumentation databases (DPV), ${ }^{2}$ the German Diabetes Versorgungs-Evaluation (DIVE), ${ }^{3}$ and others. ${ }^{4,5}$ In the past decade, the Type 1 Diabetes Exchange Clinic

LMC Diabetes and Endocrinology, Toronto, Canada.

Prior publication: This study has been presented in abstract form and presented as a poster at the 79th American Diabetes Association Annual Scientific Meeting, San Francisco, CA, June 2019.

(C) Ronnie Aronson, et al., 2020; Published by Mary Ann Liebert, Inc. This Open Access article is distributed under the terms of the Creative Commons License (http://creativecommons.org/licenses/by/4.0), which permits unrestricted use, distribution, and reproduction in any medium, provided the original work is properly credited. 
Registry (T1DX), ${ }^{6}$ an invited clinic-based cooperative, and the Optum Humedica database ${ }^{7}$ have provided similar reports in the United States. In Canada, the Canadian Study of Longevity in T1D has particularly studied adults with T1D $>50$ years. $^{8,9}$ These registries have provided meaningful snapshots of pediatric and adult T1D populations by characterizing patient demographics, management regimens, and outcomes. $^{6,10-13}$ For example, several registries have recently updated their reports to again confirm that even with recent advances in therapies and technologies, only a minority actually achieves recommended targets. $7,10,11$

An accurate and constant awareness of the clinical status of people with T1D is necessary to inform the feasibility of current guidelines and the need for future strategy redevelopment. The Canadian LMC Diabetes Registry comprises the health records of one of the largest endocrine practice groups globally, with $>50$ endocrinologists across three Canadian provinces. Since 2015 , the registry has uniquely provided quarterly real-time outcome reports, aligned to clinical guidelines, to each endocrinologist for their respective cohorts, with their centile rankings. The registry has also provided unique insights into the impact of new therapies and of novel education delivery systems and measures. ${ }^{14,15}$ This report aims to characterize the Canadian T1D patient population within the registry based on their demographics, their treatment regimens and technology utilization, and their metabolic outcomes.

\section{Methods}

\section{Study design and data source}

This study was a retrospective cohort analysis of the LMC Diabetes Registry. The LMC Diabetes Registry represents the shared electronic health records of 42,000 patients with diabetes actively attending 1 of 13 LMC clinics in Canada (10 in Ontario, 2 in Quebec, and 1 in Alberta). ${ }^{14,16}$ Patients are usually assessed quarterly by 1 of $>60$ endocrinologists and physician assistants, supported by $>50$ diabetes educators, all practicing in alignment to the Diabetes Canada Clinical Practice Guidelines, ${ }^{17}$ within a publicly funded health care system. The study was conducted in compliance with the ethical principles of the Declaration of Helsinki. An independent ethics committee approved the protocol and patients provided consent for their medical data to be used for research purposes (NCT04162067).

Individuals $\geq 18$ years of age were included in the analysis if they had a clinical diagnosis of T1D, had a visit between July 1, 2015 and June 30, 2018, and were followed by an endocrinologist for $>6$ months. All data used for analysis were the most recently available up to June 30, 2019.

Sociodemographic information included age, gender, duration at LMC, ethnicity, education level, household income, health benefits, marital, and employment status.

\section{Outcomes}

Outcomes included hemoglobin A1C (HbA1c), fasting plasma glucose, low-density lipoprotein cholesterol (LDL-C), high-density lipoprotein (HDL) cholesterol, nonHDL cholesterol, triglycerides, estimated glomerular filtration rate (eGFR), urine albumin-to-creatinine ratio (uACR), weight, body mass index (BMI), waist circumference, blood pressure, and hypoglycemia.

Hypoglycemia frequency is collected at each patient visit using a structured interview by a trained nonphysician interviewer. Patients report their frequency of "any hypoglycemia" (symptomatic and/or confirmed) in the prior week, as well as severe hypoglycemia, defined as requiring the assistance of another person, within the prior year.

At each visit, any insulin pump, continuous glucose monitoring (CGM) device, and self-measured blood glucose (SMBG) meter brands were recorded and the device-stored data were uploaded $\left(\right.$ Glooko $\left.^{\circledR}\right)$ or shared $\left(\right.$ Clarity ${ }^{\circledR}$, CareLink $^{\mathrm{TM}}$ or LibreView $\left.{ }^{\circledR}\right)$.

A microvascular condition was defined as a clinical diagnosis of neuropathy, retinopathy, or nephropathy (also defined as uACR $\geq 2.0 \mathrm{mg} / \mathrm{mmol}$ or eGFR $<60 \mathrm{~mL} / \mathrm{min} /$ $1.73 \mathrm{~m}^{2}$ ). A macrovascular condition was defined as the presence of either coronary, cerebral, or peripheral vascular disease or revascularization. A mental health condition was defined as a clinical diagnosis or the use of a prescription antidepressant, antianxiety, or antipsychotic therapy.

CGM or flash glucose monitoring (FGM) outcomes included mean glucose, standard deviation (SD), coefficient of variation, time in range (TIR, 3.9 to $10.0 \mathrm{mmol} / \mathrm{L}$ ), time above range $(>10.0 \mathrm{mmol} / \mathrm{L})$, and time below range $(<3.9 \mathrm{mmol} / \mathrm{L})$. The most recently available CGM data were used, using a minimum of 5 days and a maximum of 90 days, where the active status of the device was $>70 \%$.

Individuals were considered as "carbohydrate counting", for bolus dosing if a carbohydrate count was recorded for $>50 \%$ of their total bolus dose volume, over the prior 7 days. Daily SMBG frequency was assessed in a randomly selected one-third subgroup of non-CGM/FGM users, over their prior 14 days of SMBG data.

\section{Statistical analyses}

All demographic and clinical data were tabulated by age cohort (18-25 years, 26 to 49 years, $\geq 50$ years). Continuous variables are presented as mean \pm SD and categorical variables are presented as percentages. The main variables of interest for comparison between age cohorts, and between multiple daily injections (MDI) versus continuous subcutaneous insulin infusion (CSII) users, were $\mathrm{HbA1c}$, weight, BMI, LDL-C, blood pressure, hypoglycemia, and CGM metrics. Multivariable linear regression was used to determine differences between age cohorts in $\mathrm{HbA} 1 \mathrm{c}$, weight, BMI, LDL-C, blood pressure, and continuous CGM metrics, adjusting for duration of diabetes and type of insulin therapy (MDI vs. CSII). The model for HbAlc was also adjusted for CGM/FGM use, the model for LDL-C was additionally adjusted for use of lipid lowering therapy, the models for blood pressure were additionally adjusted for use of antihypertensive therapy, and the models for CGM metrics were also adjusted for device type (CGM or FGM). Multivariable regression was used to test HbA1c differences in SMBG frequency $<2$ versus $\geq 2 /$ day (adjusting for age, diabetes duration, and mode of insulin therapy); and in carbohydrate counting $<20 \%$ and $\geq 50 \%$ of bolus dosing, (adjusting for age and duration of diabetes). Multivariable logistic regression was used to evaluate differences between age cohorts in the proportions of individuals with $\mathrm{HbA} 1 \mathrm{c} \leq 7.0 \%$ and $>9.0 \%$, 
those with LDL-C $\geq 2.0 \mathrm{mmol} / \mathrm{L}$, and those reporting hypoglycemia and severe hypoglycemia. Similar multivariable linear regression models and multivariable logistic regression models were used to assess differences in clinical outcomes between MDI and CSII users, also adjusting for age. Differences between age cohorts in the proportion of individuals using CSII therapy, and a CGM/FGM device, were evaluated with a chi-square test. All regression models that compared the three age cohorts used a Tukey-Kramer adjustment to account for multiple comparisons.

Missing data were not replaced. All analyses were conducted with SAS v. 9.4 (SAS Institute, Inc., Cary, NC). A $P$-value $<0.05$ was considered statistically significant.

\section{Results}

Of the 5113 patients with T1D in the LMC Diabetes Registry, 4107 had a visit with an endocrinologist between July 1, 2015 and June 30, 2018, and 3600 had been followed by an endocrinologist for $\geq 6$ months and had given prior consent for their data to be used, forming the final cohort. Their mean age was $43.9 \pm 15.3$ years, with an age range of 18-92 years and mean duration of diabetes was $21.5 \pm 13.9$ years (Table 1). Males made up 55\%, 80\% identified as Caucasian, and 53\% reported a family history of diabetes. Half were married and $85.4 \%$ were employed or studying. In the cohort, $39.4 \%$ were using CSII therapy $(n=1417)$ and were numerically younger, and had been in care at LMC longer, compared with the MDI cohort ( $n=2183$, Supplementary Table S1).

\section{HbA1c outcomes}

Metabolic outcomes of the total cohort and by age cohort are presented in Table 2. The most recent HbA1c mean was $8.1 \% \pm 1.5 \%$ and $22.5 \%$ had achieved the target $\mathrm{HbA} 1 \mathrm{c} \leq 7.0 \%$. Mean HbA1c was highest, and the proportion with HbA1c $\leq 7.0 \%$ was lowest, in the $18-25$ age cohort $(P<0.01)$ (Fig. 1a). The proportion of individuals with $\mathrm{HbA1c}>9.0 \%$ was lowest in the $\geq 50$ cohort $(14.0 \%)$ compared with the 18-25 age cohort $(35.1 \%)(P<0.001)$ and the $26-49$ age cohort $(21.9 \%)$ $(P<0.001)$. When examined by ethnicity, the highest HbA1c levels were seen among the non-Hispanic black cohort with a mean of $9.0 \% \pm 2.1 \%$ versus $8.3 \% \pm 1.6 \%$ for the Asian and $8.0 \% \pm 1.4 \%$ for non-Hispanic white populations.

There was a significant interaction between mode of insulin delivery and CGM use $(P<0.01)$. MDI only was associated with a significantly higher HbA1c compared with pump only, MDI+CGM, and pump+CGM $(P<0.001)$ (Fig. 1b). In individuals not using a CGM/FGM device, pump users showed a lower mean HbA1c than MDI users (CSII $7.8 \% \pm 0.1 \%$ vs. MDI $8.4 \% \pm 0.1 \%, P<0.001$ ). Among CGM/FGM users, pump users continued to show a smaller, but statistically significant, HbA1c benefit (CSII 7.8\% $\pm 0.1 \%$ vs. MDI $8.0 \% \pm 0.1 \%, P=0.03)$. This pattern of results was consistent in the 26-49 and $\geq 50$ age cohorts $(P<0.01)$.

Among MDI users, use of CGM/FGM was associated with a lower HbA1c $(8.4 \% \pm 0.1 \%$ vs. $8.0 \% \pm 0.1 \%, P<0.001)$. Among pump users, the mean $\mathrm{HbA} 1 \mathrm{c}$ was $7.8 \%$ regardless of CGM/FGM use $(P=0.79)$.

Among 524 individuals with pump data available, there was a mean of $5.0 \pm 2.4$ bolus insulin doses administered/day, and a carbohydrate count was entered 3.2 1.6 times/day. Carbohydrate counting (defined as a carbohydrate entry associated with $>50 \%$ of bolus dose volume) was associated with a lower HbA1c $(7.7 \% \pm 0.9 \%$ vs. $8.2 \% \pm 1.2 \%$, $P<0.01)$. Individuals who "rarely" carb counted $(<20 \%$ of their bolus insulin dosing) were few $(n=35)$ but showed no difference in HbA1c versus "regularly" carb counters $(8.0 \% \pm 1.6 \%$ vs. $7.7 \% \pm 1.0 \%$, respectively, $P=0.29)$. Highest mean HbA1c was seen in the group who could be seen as using carbohydrate counting intermittently (associated with $20 \%-50 \%$ of their bolus doing) at $8.6 \% \pm 1.3 \%$ (Supplementary Table S2).

Among those not using CGM/FGM, a randomly selected subgroup of 495 patients showed a mean daily SMBG frequency of $3.4 \pm 2.2$ (median 3.3). CSII users utilized SMBG more frequently $(4.0 \pm 3.7)$ compared with MDI users $(2.9 \pm 2.1)$. Overall, a higher mean SMBG frequency was associated with a lower mean HbA1c $(r=-0.30)$. A mean testing frequency of $<2$ times/day was associated with a higher $\mathrm{HbA} 1 \mathrm{c}(8.4 \% \pm 0.1 \%)$ compared with a mean testing frequency of at least two or more times/day $(7.8 \% \pm 0.1 \%)$ $(P<0.001)$, adjusted for age, duration of T1D, and mode of insulin delivery (Supplementary Table S3).

\section{Other metabolic outcomes}

In the total cohort, mean weight was $79.6 \pm 18.2 \mathrm{~kg}$, and $63.3 \%$ had a BMI $>25 \mathrm{~kg} / \mathrm{m}^{2}$ (Table 2). Weight and BMI were both significantly higher in the two older age cohorts compared with the $18-25$ age cohort $(P<0.05)$. There were no differences in weight $(P=0.37)$ or BMI $(P=0.35)$ between MDI and CSII users (Supplementary Table S4).

Mean LDL-C was $2.3 \pm 0.8 \mathrm{mmol} / \mathrm{L}$. Statin use in the overall cohort was $44.5 \%$ but among those $>30$ years of age and with diabetes duration $>15$ years, $62.7 \%$ were using statin therapy.

Mean systolic blood pressure was $121.6 \pm 15.3 \mathrm{mmHg}$ (Table 2) and $60.9 \%$ of the cohort had achieved a blood pressure of $<130 / 80 \mathrm{mmHg}$. Systolic blood pressure increased with age $(P<0.001)$ and antihypertensive therapy was more prevalent with increasing age (18-25 age cohort 4.6\%; 26-49 age cohort $26.0 \%$; $\geq 50$ age cohort $68.7 \%$ (Table 3 ).

\section{Hypoglycemia}

For any hypoglycemia, $63.1 \%$, reported at least one event weekly and the proportion trended lower from the youngest to oldest (18-25 age cohort $67.0 \%$; 26-49 age cohort $63.5 \%$; $\geq 50$ age cohort $61.2 \%$ ) reaching significance in the $\geq 50$ age cohort $(P<0.05)$. The mean incidence of self-reported weekly hypoglycemia was $1.2 \pm 2.2$ and similarly trended downward with advancing age group $(1.5 \pm 0.1 ; 1.3 \pm 0.1$; and $1.1 \pm 0.1$, respectively; $P<0.05)$. For severe hypoglycemia, $3.6 \%$ of patients reported at least one event in the prior year and this proportion did not differ across age groups $(P=0.13)$. Self-reported hypoglycemia frequency did not differ between MDI and CSII cohorts (MDI 1.2 \pm 0.1 ; CSII $1.3 \pm 0.1, P>0.05)$ but severe hypoglycemia was significantly lower in the CSII cohort $(2.6 \%)$ than the MDI cohort $(4.3 \%)(P=0.03)$.

\section{Metabolic therapies and devices}

Nearly all $(98.1 \%)$ were using analogue insulins. Individuals using CSII therapy (39.3\%) were numerically younger, and had been in care at LMC longer, compared with 
Table 1. Participant Characteristics

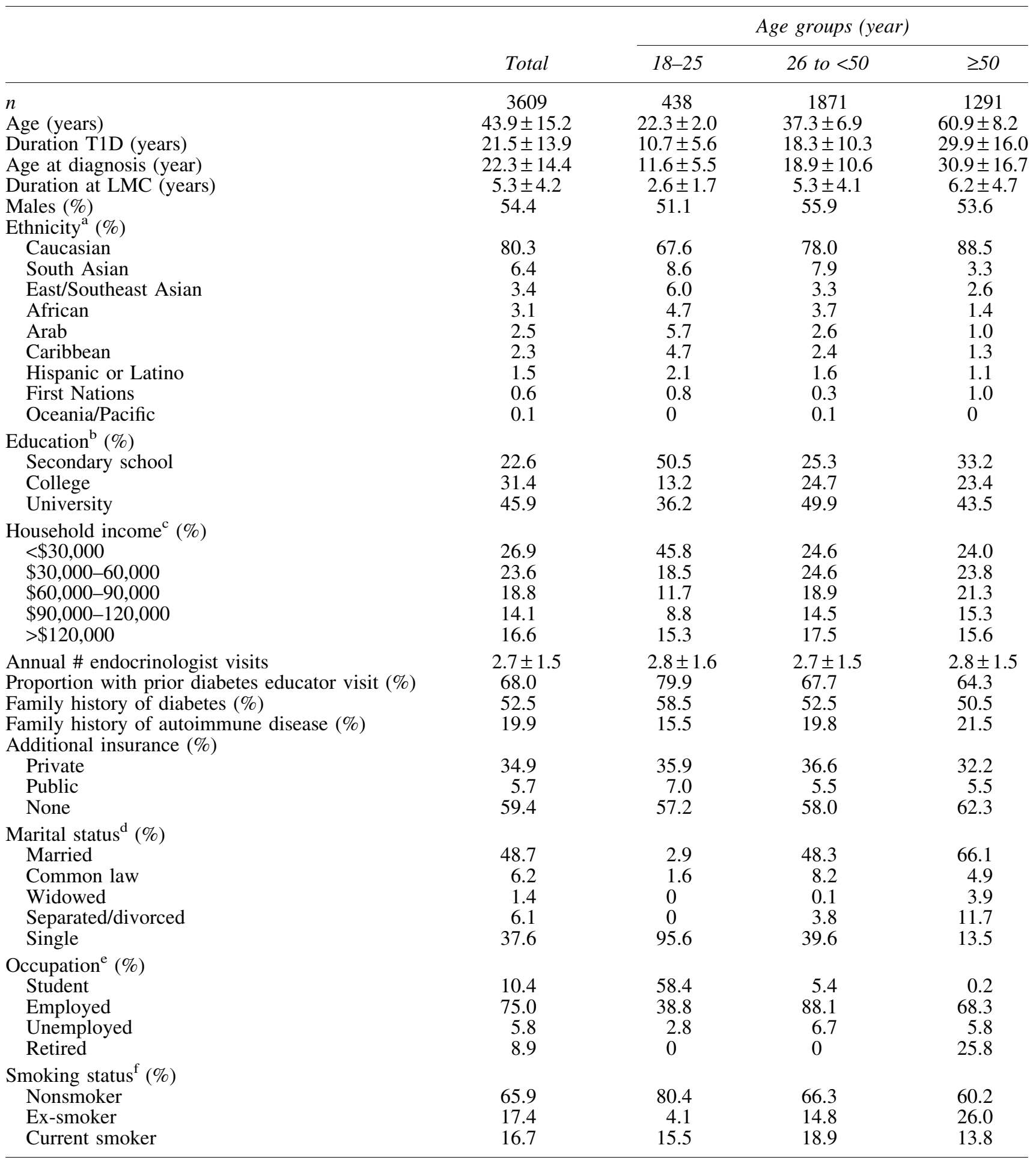

${ }^{\mathrm{a}} n=3014$ for ethnicity; ${ }^{\mathrm{b}} n=2526$ for education; ${ }^{\mathrm{c}} n=2086$ for household income; ${ }^{\mathrm{d}} n=3076$ for marital status; ${ }^{\mathrm{e}} n=3106$ for occupation; ${ }^{\mathrm{f}} n=3111$ for smoking status.

T1D, type 1 diabetes.

the MDI cohort (60.7\%, Table 3, Supplementary Table S5). Insulin pump use was lower with increasing age (18-25 age cohort $43.5 \%$; $26-49$ age cohort $42 \%$; $\geq 50$ age cohort $36.1 \%$; $P<0.001)$. Insulin pump use was also lowest among the black population at $24.2 \%$ versus $36.5 \%$ among Asians and $43.2 \%$ among non-Hispanic whites. Of the total cohort, $22.7 \%$ were using or had used a wearable glucose sensor device (CGM $6.1 \%$; FGM 16.6\%) and was more prevalent with increasing age $(16.4 \%, 23.4 \%$, and $23.8 \%$, respectively; $P<0.01)$. CSII users were more likely to also be using a CGM/FGM device $(27.1 \%)$ compared with the MDI cohort $(19.8 \%)(P<0.01)$ (Supplementary Table S5). 
Table 2. Metabolic Status of Type 1 Diabetes Registry

\begin{tabular}{|c|c|c|c|c|c|c|c|c|}
\hline & \multirow[b]{2}{*}{$\mathrm{n}$} & \multirow[b]{2}{*}{ Total } & \multicolumn{3}{|c|}{ Age cohorts (years) } & \multicolumn{3}{|c|}{ Ethnicity } \\
\hline & & & $18-25$ & 26 to $<50$ & $\geq 50$ & $\begin{array}{l}\text { Non- } \\
\text { Hispanic } \\
\text { white }\end{array}$ & Asian & $\begin{array}{l}\text { Non- } \\
\text { Hispanic } \\
\text { black }\end{array}$ \\
\hline $\begin{array}{l}\text { A1C }(\%) \\
\leq 7.0 \% \\
7.1 \% \text { to } \leq 8.0 \% \\
8.1 \% \text { to } \leq 9.0 \% \\
>9.0 \%\end{array}$ & 3524 & $\begin{array}{c}8.1 \pm 1.5 \\
22.5 \\
33.2 \\
23.7 \\
20.8\end{array}$ & $\begin{array}{c}8.6 \pm 1.8 \\
17.1 \\
27.7 \\
20.1 \\
35.1\end{array}$ & $\begin{array}{c}8.1 \pm 1.5 \\
22.6 \\
32.4 \\
23.1 \\
21.9\end{array}$ & $\begin{array}{l}7.9 \pm 1.2 \\
24.2 \\
36.1 \\
25.7 \\
14.0\end{array}$ & $\begin{array}{c}8.0 \pm 1.4 \\
23.1 \\
34.7 \\
23.8 \\
18.5\end{array}$ & $\begin{array}{c}8.3 \pm 1.6 \\
22.7 \\
26.5 \\
24.4 \\
26.5\end{array}$ & $\begin{array}{c}9.0 \pm 2.1 \\
15.6 \\
24.0 \\
24.0 \\
36.4\end{array}$ \\
\hline $\begin{array}{l}\text { Fasting plasma } \\
\text { glucose }(\mathrm{mmol} / \mathrm{L})\end{array}$ & 2621 & $9.4 \pm 3.4$ & $9.9 \pm 4.8$ & $9.4 \pm 4.3$ & $9.1 \pm 4.0$ & $9.3 \pm 4.2$ & $9.1 \pm 3.8$ & $9.3 \pm 4.7$ \\
\hline $\begin{array}{l}\mathrm{LDL}-\mathrm{C}(\mathrm{mmol} / \mathrm{L}) \\
\leq 2.0 \mathrm{mmol} / \mathrm{L}(\%)\end{array}$ & 3307 & $\begin{array}{l}2.3 \pm 0.8 \\
42.4\end{array}$ & $\begin{array}{c}2.4 \pm 0.7 \\
31.3\end{array}$ & & $\begin{array}{c}2.0 \pm 0.7 \\
61.5\end{array}$ & $\begin{array}{c}2.2 \pm 0.8 \\
45.0\end{array}$ & $\begin{array}{c}2.4 \pm 0.9 \\
35.1\end{array}$ & $\begin{array}{c}2.5 \pm 0.9 \\
28.0\end{array}$ \\
\hline $\begin{array}{l}\text { olesterol } \\
1 / \mathrm{L})\end{array}$ & 3345 & $1.5 \pm 0.4$ & $1.5 \pm 0.5$ & $1.5 \pm 0.5$ & $1.6 \pm 0.6$ & $1.6 \pm 0.5$ & $1.5 \pm 0.5$ & $1.5 \pm 0.4$ \\
\hline $\begin{array}{l}\text { Non-HDL } \\
\text { cholesterol } \\
\text { (mmol/L) }\end{array}$ & 3029 & $2.8 \pm 0.9$ & $2.9 \pm 0.9$ & $2.9 \pm 1.0$ & $2.4 \pm 0.7$ & $2.7 \pm 0.9$ & $2.9 \pm 0.9$ & $3.0 \pm 1.0$ \\
\hline $\begin{array}{l}\text { Triglycerides } \\
(\mathrm{mmol} / \mathrm{L})\end{array}$ & 3246 & $1.1 \pm 0.8$ & $1.1 \pm 0.7$ & $1.2 \pm 1.0$ & $1.1 \pm 0.6$ & $1.1 \pm 0.7$ & $1.2 \pm 1.2$ & $1.3 \pm 1.3$ \\
\hline eGFR (\%) & 3277 & & & & & & & \\
\hline $\begin{array}{l}<30 \\
30 \text { to }<60\end{array}$ & & $\begin{array}{l}2.0 \\
5.9\end{array}$ & $\begin{array}{l}0 \\
0\end{array}$ & $\begin{array}{l}1.4 \\
3.0\end{array}$ & $\begin{array}{r}3.5 \\
12.1\end{array}$ & $\begin{array}{l}2.0 \\
6.3\end{array}$ & $\begin{array}{l}1.1 \\
1.8\end{array}$ & $\begin{array}{l}3.4 \\
4.8\end{array}$ \\
\hline$\geq 60$ & & 92.1 & 100.0 & 95.6 & 84.4 & 91.7 & 97.1 & 91.8 \\
\hline $\begin{array}{l}\text { uACR }(\%) \\
\geq 2.0\end{array}$ & 2921 & 27.6 & 22.8 & 24.7 & 33.2 & 27.6 & 24.9 & 40.2 \\
\hline $\begin{array}{l}\text { Weight }(\mathrm{kg}) \\
\text { BMI }\left(\mathrm{kg} / \mathrm{m}^{2}\right) \\
\quad<18.5 \\
18.5 \text { to }<25.0 \\
25.0 \text { to }<30 \\
\geq 30.0\end{array}$ & $\begin{array}{l}3527 \\
3521\end{array}$ & $\begin{array}{c}79.6 \pm 18.2 \\
27.4 \pm 5.6 \\
1.4 \\
35.4 \\
37.1 \\
26.2\end{array}$ & $\begin{array}{c}74.0 \pm 15.3 \\
25.4 \pm 4.6 \\
2.5 \\
50.5 \\
32.0 \\
15.0\end{array}$ & $\begin{array}{c}81.1 \pm 18.6 \\
27.7 \pm 5.7 \\
1.2 \\
33.5 \\
37.4 \\
27.9\end{array}$ & $\begin{array}{c}79.3 \pm 18.1 \\
27.7 \pm 5.8 \\
1.2 \\
33.0 \\
38.3 \\
27.5\end{array}$ & $\begin{array}{c}80.6 \pm 18.0 \\
27.6 \pm 5.6 \\
0.9 \\
34.2 \\
38.0 \\
26.8\end{array}$ & $\begin{array}{c}71.7 \pm 15.5 \\
25.7 \pm 4.7 \\
2.4 \\
47.4 \\
32.8 \\
17.4\end{array}$ & $\begin{array}{c}81.6 \pm 21.1 \\
28.3 \pm 7.6 \\
5.1 \\
30.6 \\
29.3 \\
35.0\end{array}$ \\
\hline $\begin{array}{l}\text { Waist } \\
\text { circumference } \\
(\mathrm{cm})\end{array}$ & 3129 & $93.2 \pm 14.7$ & $85.8 \pm 11.6$ & $93.3 \pm 14.8$ & $95.6 \pm 14.5$ & $94.0 \pm 14.5$ & $88.8 \pm 12.5$ & $93.7 \pm 16.5$ \\
\hline $\begin{array}{l}\text { Systolic BP } \\
\text { (mmHg) }\end{array}$ & 3563 & $121.7 \pm 15.2$ & $114.9 \pm 13.4$ & $119.7 \pm 14.1$ & $126.9 \pm 15.8$ & $122.2 \pm 15.2$ & $114.9 \pm 14.2$ & $121.6 \pm 15$. \\
\hline $\begin{array}{l}\text { Diastolic BP } \\
(\mathrm{mmHg})\end{array}$ & 3571 & $72.6 \pm 9.3$ & $71.7 \pm 9.2$ & $73.7 \pm 9.4$ & $71.4 \pm 9.1$ & $72.5 \pm 9.1$ & $70.1 \pm 9.5$ & $75.5 \pm 10.1$ \\
\hline $\mathrm{BP}<130 / 80 \mathrm{mmHg}$ & & 60.9 & 72.9 & 63.5 & 53.2 & 60.4 & 76.6 & 56.6 \\
\hline
\end{tabular}

Data are presented as mean $\pm \mathrm{SD}$, or as a percentage.

BMI, body mass index; eGFR, estimated glomerular filtration rate; HDL, high-density lipoprotein; LDL-C, low-density lipoprotein cholesterol; SD, standard deviation; uACR, urine albumin-to-creatinine ratio.

Adjunct antihyperglycemic therapy was used in $11.9 \%$ of the patient cohort, with metformin $(6.2 \%)$ and SGLT2 inhibitors $(5.5 \%)$ used commonly (Table 3 ). Adjusted mean HbA1c was slightly lower among individuals using adjunct antihyperglycemic therapy $(8.0 \% \pm 0.1 \%$ vs. $8.2 \% \pm 0.1 \%)$.

\section{Comorbidities}

A microvascular condition was present in $34.9 \%$ of the cohort (Table 3). Nephropathy was present in $26.8 \%$, retinopathy in $14.1 \%$, and clinically significant neuropathy in $6.5 \%$. Erectile dysfunction was reported in $4.9 \%$. Each disorder was increasingly prevalent with advancing cohort age. Macrovascular disease was less common, affecting $4.8 \%$ of the overall cohort but almost entirely affecting the age $\geq 50$ cohort where $8.8 \%$ had a history of coronary artery disease, $2.2 \%$ had cerebral vascular disease, and $1.6 \%$ had peripheral vascular disease. There was a slightly higher prevalence of microvascular conditions in MDI users (36.4\%) than CSII users $(32.6 \%)(P=0.04)$ and no difference in prevalence of macrovascular conditions (Supplementary Table S6). In the total cohort, $15.9 \%$ had a diagnosed and/or treated mental health condition, with a prevalence of nearly $20 \%$ in the $\geq 50$ age cohort (Table 3 ).

\section{CGM/FGM data}

Of the total cohort, $22.7 \%(n=816)$ were using a CGM/ FGM device. Data from 623 individuals were available (68.6\% FreeStyle Libre, 31.4\% Dexcom), with a mean of $64.7 \pm 32.1$ days of data (within the prior 5 to 90 days) and with $91.4 \% \pm 8.6 \%$ active CGM time (Supplementary Table S7). In the total cohort, mean TIR was $53.4 \% \pm 17.0 \%$, mean CGM/FGM glucose was $9.6 \pm 2.0 \mathrm{mmol} / \mathrm{L}$ and only 

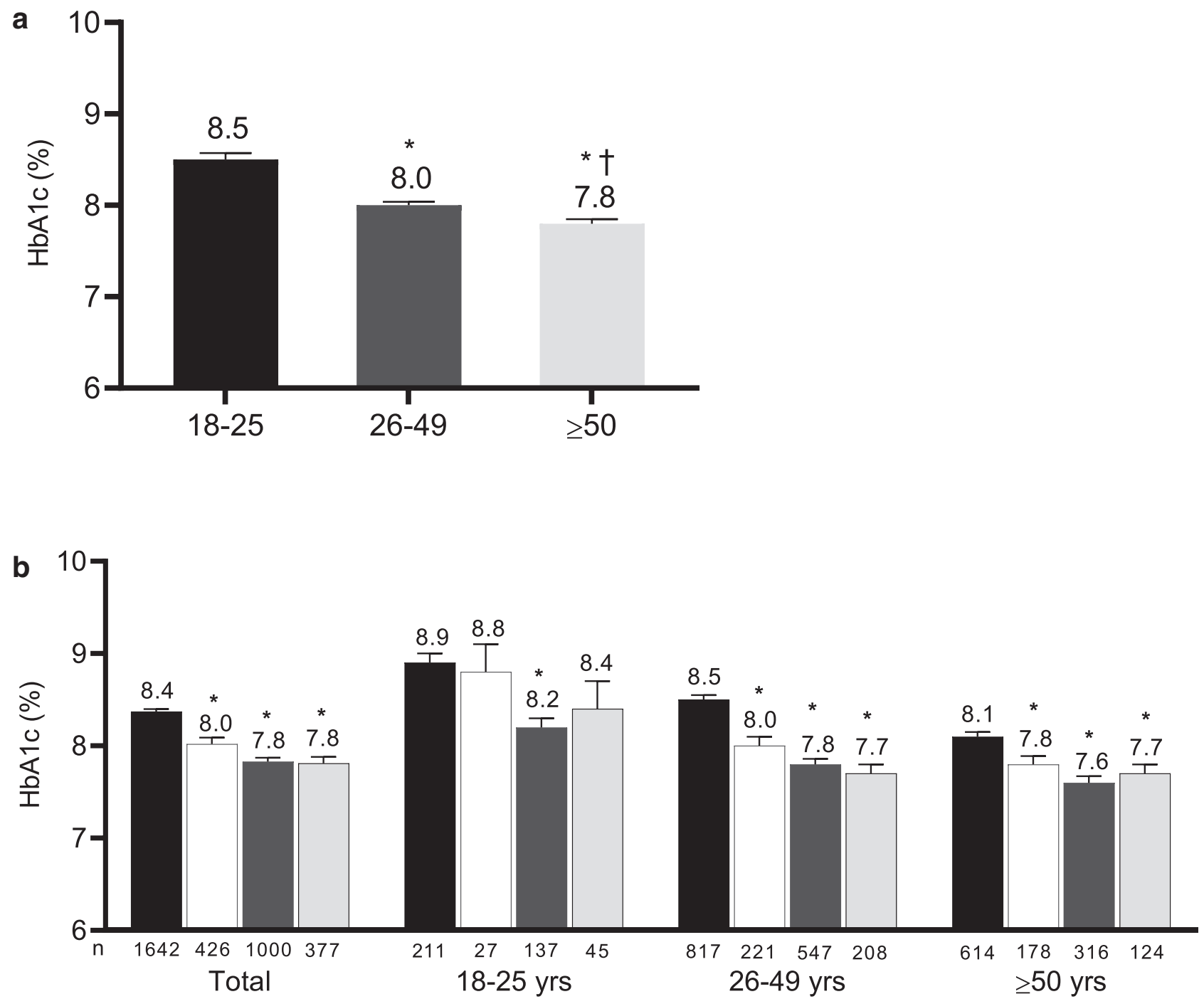

FIG. 1. (a) Metabolic outcomes by age cohort. Data are presented as mean and standard error. *Significantly different compared with the $18-25$ age cohort $(P<0.05)$. ${ }^{\dagger}$ Significantly different compared with the $\geq 50$ age cohort $(P<0.05)$. (b) $\mathrm{HbA1c}$ stratified by patients using MDI/CSII therapy and/or a CGM/FGM device. Data are presented as mean and standard error. Black bars = MDI only; white bars = MDI+CGM; dark gray bars=CSII only, light gray bars = CSII+CGM. *Significantly different compared with MDI only $(P<0.05)$. CSII, continuous subcutaneous insulin infusion; CGM, continuous glucose monitor; FGM, flash glucose monitoring; HbA1c, hemoglobin A1C; MDI, multiple daily injections.

$36.3 \%$ showed $\mathrm{CV}<36 \%$. Time above range was $40.3 \% \pm$ $18.7 \%$ and time below range was $6.3 \% \pm 5.9 \%$. The $18-25$ age cohort showed the highest time below range and highest variability compared with both older cohorts $(P<0.05)$ (Supplementary Table S7). There were no significant differences in CGM-measured outcomes between CSII and MDI users (Supplementary Table S8). Finally, across all CGM/FGM users, CGM users showed a higher TIR (58.8\% \pm $18.5 \%$ vs. $51.0 \% \pm 15.8 \%, P<0.001)$, lower variability (SD: $3.4 \pm 0.8$ vs. $3.9 \pm 1.0, P<0.001$ ), (Supplementary Table S9).

\section{Discussion}

This study reports on the current state and management of a large Canadian cohort of 3600 adults with T1D and provides comparisons across different age cohorts and different insulin delivery modes. Majority of these adults with T1D did not meet metabolic targets, particularly if they were younger. The use of advanced technologies has also remained relatively low.

Among all individuals, only $22 \%$ had achieved the American Diabetes Association ${ }^{18}$ and Diabetes Canada ${ }^{17}$ recommended target $\mathrm{HbA} 1 \mathrm{c}$ of $\leq 7.0 \%$. These results are virtually identical to the latest data from the T1DX, which reported that $21 \%$ of U.S. adults had an $\mathrm{HbA} 1 \mathrm{c} \leq 7.0 \%{ }^{10}$ Interestingly, T1DX also showed little change in $\mathrm{HbA1c}$ levels between 2010 and 2018, despite increases in insulin pump and CGM use. Our finding of higher HbA1c levels among younger age groups have similarly been reported in earlier registry reports. ${ }^{11,19,20}$

One of the unique elements in this registry is that each endocrinologist receives a quarterly report outlining their patient populations' achievements in metabolic outcomes, expressed both as means and as centile ranking within the 
Table 3. Therapies, Devices, and Comorbidities in the Type 1 Diabetes Registry Cohort

\begin{tabular}{|c|c|c|c|c|c|c|c|}
\hline & \multirow[b]{2}{*}{ Total } & \multicolumn{3}{|c|}{ Age cohorts (years) } & \multicolumn{3}{|c|}{ Ethnicity } \\
\hline & & $18-25$ & 26 to $<50$ & $\geq 50$ & $\begin{array}{c}\text { Non-Hispanic } \\
\text { white }\end{array}$ & Asian & $\begin{array}{c}\text { Non-Hispanic } \\
\text { black }\end{array}$ \\
\hline$n$ & 3600 & 438 & 1871 & 1291 & 2419 & 296 & 161 \\
\hline \multirow{2}{*}{\multicolumn{8}{|c|}{$\begin{array}{l}\text { Therapies } \\
\text { Type of insulin therapy (\%) }\end{array}$}} \\
\hline & & & & & & & \\
\hline MDI & 60.7 & 56.5 & 58.0 & 63.9 & 56.9 & 63.5 & 75.8 \\
\hline CSII & 39.3 & 43.5 & 42.0 & 36.1 & 43.2 & 36.5 & 24.2 \\
\hline Adjunct antihyperglycemic therapies (\%) & 12.0 & 4.8 & 12.2 & 14.1 & 11.4 & 12.2 & 11.8 \\
\hline Metformin & 6.2 & 3.4 & 5.7 & 7.8 & 5.3 & 7.8 & 10.6 \\
\hline SGLT2i & 5.5 & 1.4 & 5.6 & 6.7 & 5.5 & 4.1 & 4.4 \\
\hline GLP-1 RA & 1.9 & 0.2 & 2.4 & 1.8 & 1.8 & 1.4 & 1.2 \\
\hline DPP-4i & 1.3 & 0.2 & 1.3 & 1.6 & 0.9 & 0.3 & 4.4 \\
\hline Glucagon availability, $n(\%)$ & 26.9 & 40.0 & 26.2 & 23.3 & 26.9 & 32.8 & 28.6 \\
\hline Lipid lowering therapy (statin) (\%) & 44.5 & 1.4 & 32.3 & 76.7 & 46.5 & 37.5 & 30.4 \\
\hline Antihypertensive therapy (\%) & 38.7 & 4.6 & 26.0 & 68.7 & 40.6 & 24.7 & 36.0 \\
\hline RAAS inhibitors $(\%)$ & 36.3 & 4.3 & 24.4 & 64.1 & 38.2 & 24.0 & 32.3 \\
\hline \multicolumn{8}{|l|}{ Diabetes devices } \\
\hline \multicolumn{8}{|l|}{ Pump model in patients using CSII (\%) } \\
\hline Medtronic ${ }^{\mathrm{TM}}$ & 61.6 & 63.8 & 58.4 & 66.2 & 62.6 & 59.3 & 48.7 \\
\hline OmniPod $^{\mathrm{TM}}$ & 26.1 & 24.3 & 28.4 & 23.1 & 25.3 & 26.9 & 46.2 \\
\hline Animas $^{\mathrm{TM}}$ & 10.5 & 11.9 & 11.0 & 9.0 & 10.5 & 11.1 & 5.1 \\
\hline Tandem ${ }^{\mathrm{TM}}$ & 1.2 & 0 & 1.6 & 1.1 & 1.1 & 1.9 & 0 \\
\hline Accu-chek ${ }^{\mathrm{TM}}$ & 0.6 & 0 & 0.8 & 0.7 & 0.6 & 0.9 & 0 \\
\hline CGM/FGM (\%) & 22.7 & 16.4 & 23.4 & 23.8 & 23.7 & 25.0 & 17.4 \\
\hline \multicolumn{8}{|c|}{ CGM/FGM model in patients using CGM/FGM (\%) } \\
\hline FreeStyle Libre & 73.2 & 83.3 & 70.3 & 74.9 & 71.7 & 71.6 & 96.4 \\
\hline Dexcom & 22.7 & 15.3 & 25.9 & 19.9 & 23.0 & 24.3 & 3.6 \\
\hline Medtronic & 4.2 & 1.4 & 3.9 & 5.3 & 5.3 & 4.1 & 0 \\
\hline \multicolumn{8}{|l|}{ Comorbidities } \\
\hline Microvascular disorders $(\%)$ & 34.9 & 20.6 & 29.6 & 47.4 & 34.1 & 31.4 & 38.5 \\
\hline Nephropathy & 26.8 & 20.6 & 21.9 & 31.6 & 27.3 & 25.0 & 34.2 \\
\hline Retinopathy & 14.1 & 2.5 & 12.0 & 21.2 & 15.6 & 11.5 & 12.4 \\
\hline Neuropathy & 6.5 & 0.7 & 4.3 & 11.6 & 6.6 & 4.1 & 6.2 \\
\hline Macrovascular disorders (\%) & 4.8 & 0.2 & 1.6 & 11.1 & 5.3 & 3.0 & 3.1 \\
\hline Coronary artery disease & 3.7 & 0.2 & 1.0 & 8.8 & 4.1 & 3.0 & 2.5 \\
\hline Cerebral vascular disease & 1.0 & 0 & 0.4 & 2.2 & 1.1 & 0.3 & 0.6 \\
\hline Peripheral vascular disease & 0.7 & 0 & 0.2 & 1.6 & 0.7 & 0.0 & 1.2 \\
\hline Mental health condition (\%) & 15.9 & 10.7 & 14.6 & 19.6 & 17.8 & 5.7 & 11.2 \\
\hline Males with erectile dysfunction (\%) & 8.9 & 0.5 & 7.0 & 14.6 & & & \\
\hline
\end{tabular}

All data are presented as a percentage, unless otherwise specified.

Nephropathy was defined as a clinical diagnosis of nephropathy or microalbuminuria, or a most recent $\mathrm{uACR} \geq 2.0 \mathrm{mg} / \mathrm{mmol}$ or eGFR $<60 \mathrm{~mL} / \mathrm{min} / 1.73 \mathrm{~m}^{2}$. Neuropathy and retinopathy were based on clinical diagnoses. Coronary artery disease was defined as presence of angina, cardiovascular disease, congestive heart failure, coronary vascular disease, myocardial infarction, coronary artery bypass grafting or angioplasty; cerebral vascular disease was defined as history of stroke or cerebral vascular accident; peripheral vascular disease was defined as history of atherosclerotic disease, intermittent claudication, aorto-femoral bypass or femoral popliteal bypass. A mental health condition was defined as a history of anxiety, depression, bipolar affective disorder, schizophrenia or an eating disorder, or usage of an antidepressant, antianxiety, or antipsychotic therapy. Erectile dysfunction was defined as history of erectile dysfunction or usage of erectile dysfunction therapy.

CSII, continuous subcutaneous insulin infusion; CGM, continuous glucose monitor; DPP-4i, dipeptidyl peptidase-4 inhibitors; FGM, flash glucose monitor; GLP-1 RA, glucagon-like peptide-1 receptor agonists; MDI, multiple daily injections; RAAS, renin angiotensin aldosterone system; SGLT2i, sodium glucose co-transporter 2 inhibitors.

group, along with the proportion using evidence-based cardiovascular disease prevention therapies. In other jurisdictions, ${ }^{21}$ quality improvement initiatives have similarly involved outcomes reporting for pediatric T1D populations. In a comparison of pediatric outcomes among eight high income countries, optimal glycemic outcomes appeared to be achievable where specific center performance data were publicly accessible, and where professional development initiatives were additionally targeted to results. In adult health care, although tempting to progress beyond individual physician feedback reporting, the transparent publication of performance data has not been consistently effective, at least in hospital-based care. ${ }^{22}$ Novel approaches are still needed.

Lower HbA1c in CSII users has also been reported in clinical trials ${ }^{23,24}$ as well in the T1DX, DIVE, and DPV registries. ${ }^{10,11}$ Insulin pump use in T1D varies significantly by country, with pump use in adults $\geq 25$ years as low as $1 \%$ in Latvia and as high as $83 \%$ in Austria. ${ }^{13}$ In this Canadian 
cohort, only $40 \%$ were using CSII therapy, compared with the $63 \%$ reported in the United States, ${ }^{10}$ indicating an underutilization of advanced technologies in Canada. Although our data have also shown a glycemic benefit in CSII versus MDI users, the advantage was much smaller in the subgroup using CGM, demonstrating the potential glycemic benefit of CGM in reducing the impact of the chosen mode of insulin delivery itself. However, CGM use in this cohort was also relatively low ( $22 \%$ vs. the T1DX experience of $\left.30 \%{ }^{10}\right)$ and was especially low among the 18-25 age cohort.

Many individuals were also not achieving other metabolic targets. Achievement of evidence-based targets for LDL-C and blood pressure is important to reduce the risk of cardiovascular disease and microvascular complications. ${ }^{17} \mathrm{Ca}$ nadian clinical practice guidelines call for statin therapy for people $>30$ years of age with a diabetes duration $>15$ years ${ }^{17}$ and in this group, only $62.7 \%$ were using statin therapy. Only $61 \%$ of the total cohort had achieved target blood pressure, whereas antihypertensive therapy was only in place for $26.0 \%$ of the $26-49$ cohort and $68.7 \%$ of the $\geq 50$ age group.

Among CGM users, mean TIR was $53.4 \%$ and the recommended target of $>70 \%{ }^{25,26}$ was only achieved by $15.7 \%$ of CGM users. Mean time in hyperglycemia and hypoglycemia were also well above the recommended targets of $<25 \%$ and $<4 \%$, respectively. ${ }^{27}$ As seen in the other realworld reports, CGM/FGM users in this cohort showed no difference in glycemia between individuals using MDI versus CSII therapy. ${ }^{28}$ In this cohort, CGM users showed improved TIR and lower variability than FGM users. This difference appears to have been driven by approximately equal increases in time below range and time above range. Given the more recent introduction of FGM versus CGM, the glycemic outcomes differences may be due to considerably different respective durations of device use.

Certain self-care behaviors have become tenets in the effort to achieve optimal glycemic control. The relationship between SMBG frequency and glycemia has been well established $^{29,30}$ and testing 6-10 times daily is broadly endorsed. ${ }^{31,32}$ Similarly, carbohydrate counting has undeniable value in helping an individual achieve an optimal glycemic outcome. In this "real-world" analysis, both behaviors were similarly associated with glycemic control. However, individuals who had self-selected to "rarely" carb count did not appear to have a resulting glycemic handicap. Similarly, lower SMBG frequencies (twice daily or more) were still associated with significant $\mathrm{HbA} 1 \mathrm{c}$ benefit. Prior observational reports had not explored the benefits of SMBG frequencies below four times daily. Both of these self-care behaviors are sometimes prerequisites for funding, ${ }^{33}$ often requiring arbitrary thresholds such as $\mathrm{SMBG}>4$ times daily or advanced carbohydrate counting skill that are not fully evidence based. The resulting restrictions to access funding or programs may create unintended barriers that do not align with our preferred approach of individualizing care.

The primary strength of this study is the comprehensive nature of the LMC Diabetes Registry, including a variety of sociodemographic and clinical variables, as well as CGM/ FGM and glucose meter data. In contrast, population registries reflect a more heterogeneous group; may lack insight into education, employment, and earning status; and may not have access to individual comorbidity or hypoglycemia experience. Volunteer-based registries may also reflect vol- unteer bias at either patient or clinic levels. Finally, the LMC cohort receives care within the Canadian public health system, minimizing obstacles to access evidence-based therapies and devices.

A limitation of this study is that LMC clinics primarily care for adults, and usually by referral from primary care. Individuals who had not provided prior consent and patients seen before the specified inclusion period could not be included. The results may, therefore, not be generalizable to the broader T1D population, especially children and adolescents, and individuals in primary care alone. The period of review ended in 2018 and findings such as device usage may not generalize to current populations with T1D. The LMC Diabetes Registry is based on an electronic medical record system so that information not routinely collected could not be included. Finally, only cross-sectional data were presented. Future studies should report on the clinical state and management of individuals with T1D in Canada over time.

Despite an advanced and well-resourced medical environment, including regular feedback on each specialist's individual cohort achievements, many individuals with T1D are not able to meet guidelines-based targets using guidelinederived systems and care programs. Younger adults appear to be the most deficient, in both control and in adoption of new technology. Use of adjunct risk-lowering therapies was incomplete, while use of advanced technologies was generally low. This pattern of limited response to our traditional medical systems appears to be consistent across multiple health jurisdictions and consistent over time. Although evidence-based development of care guidelines must continue, health care providers need real-time feedback about their respective patient outcomes to assess the impact and the feasibility of guideline-directed care. Even with this critical feedback in hand, there further appears to be a need of novel interventions and innovative communications to more effectively achieve outcomes in a larger proportion of individuals under our care.

\section{Acknowledgment}

The authors thank Richa Parihar for her contributions to the literature review and helping to draft the introduction.

\section{Authors' Contributions}

R.A. and R.E.B. designed the study and conducted the literature review. R.E.B. performed the data analysis. All authors interpreted the study data. R.A. and R.E.B. wrote the first draft of the article. A.A., R.G., B.A., Z.Y., and J-F.Y. provided critical revisions to the article. All authors approved the final version of the article.

\section{Guarantor Statement}

Ronnie Aronson is the guarantor of this article and takes responsibility for the contents of this article

\section{Author Disclosure Statement}

R.A. reports research grants from Sanofi, Novo Nordisk, Janssen, AstraZeneca, Becton Dickinson Technologies, Boehringer Ingelheim, Eli Lilly, Zealand, Xeris, Medpace, Kowa, Insulet, Dexcom, Bausch Health, Tandem Diabetes, and Bayer; and consultancies/personal fees from Sanofi, 
Novo Nordisk, Janssen, AstraZeneca, Becton Dickinson Technologies, Boehringer Ingelheim, Eli Lilly, HTL Strefa, Gilead, Merck. R.E.B. has no conflicts of interest to declare. A.A. reports grants and personal fees outside the submitted study from Sanofi, Novo Nordisk, Janssen, AstraZeneca, Eli Lilly, Merck, and Senseonics; personal fees from Boehringer Ingelheim and Dexcom, and grants from Amgen, Gilead, Glaxo-Smith-Kline, JDRF, Lexicon, Pfizer, Xeris, and Zealand. R.G. reports research support from Abbott, AstraZeneca, Boehringer Ingelheim, Eli Lilly, GlaxoSmithKline, Janssen, Medtronic, Merck, Novartis, Novo Nordisk, Roche, Sanofi, and Takeda; serving on advisory panels for AstraZeneca, Boehringer Ingelheim, Eli Lilly, Janssen, Merck, Novo Nordisk, Roche, Sanofi, and Takeda; speaker bureaus for Abbott, AstraZeneca, Boehringer Ingelheim, Eli Lilly, Janssen, Merck, Novo Nordisk, Sanofi, and Servier; consulting for AstraZeneca, Boehringer Ingelheim, Eli Lilly, Janssen, Novo Nordisk, and Takeda.

\section{Funding Information}

This study was supported by grants from Sanofi and Insulet. Neither Sanofi nor Insulet was involved in the writing of the article.

\section{Supplementary Material}

Supplementary Table S1

Supplementary Table S2

Supplementary Table S3

Supplementary Table S4

Supplementary Table S5

Supplementary Table S6

Supplementary Table S7

Supplementary Table S8

Supplementary Table S9

\section{References}

1. Eliasson B, Gudbjornsdottir S: Diabetes care-improvement through measurement. Diabetes Res Clin Pr 2014;2:S291S294.

2. Grabert M, Schweiggert F, Holl RW: A framework for diabetes documentation and quality management in Germany: 10 years of experience with DPV. Comput Methods Programs Biomed 2002;69:115-121.

3. Danne T, Kaltheuner M, Koch A, et al.: ["DIabetes Versorgungs-Evaluation" (DIVE) — a national quality assurance initiative at physicians providing care for patients with diabetes]. Dtsch Med Wochenschr 2013;138:934-939.

4. Phelan H, Donaghue K, Cameron F, et al.: The Australasian diabetes data network (ADDN): first steps towards a national database resource. Int J Pediatr Endocrinol 2015;2015:O35.

5. Livingstone SJ, Looker HC, Hothersall EJ, et al.: Risk of cardiovascular disease and total mortality in adults with type 1 diabetes: Scottish Registry Linkage Study. PLoS Med 2012;9:e1001321.

6. Beck RW, Tamborlane WV, Bergenstal RM, et al.: The T1D exchange clinic registry. Endocrinol Metab 2012;97: 4383-4389.

7. Pettus JH, Zhou FL, Shepherd L, et al.: Differences between patients with type 1 diabetes with optimal and suboptimal glycaemic control: a real-world study of more than 30,000 patients in a US electronic health record database. Diabetes Obes Metab 2020;22:622-630.

8. Boulet G, Halpern EM, Lovblom LE, et al.: Prevalence of insulin pump therapy and its association with measures of glycemic control: results from the Canadian Study of Longevity in Type 1 Diabetes. Diabetes Technol Ther 2016;18:298-307.

9. Bai JW, Boulet G, Halpern EM, et al.: Cardiovascular disease guideline adherence and self-reported statin use in longstanding type 1 diabetes: results from the Canadian study of longevity in diabetes cohort. Cardiovasc Diabetol Diabetol 2016;15:1-10.

10. Foster NC, Beck RW, Miller KM, et al.: State of type 1 diabetes management and outcomes from the T1D Exchange in 2016-2018. Diabetes Technol Ther 2019;21: 66-72.

11. Van Mark G, Lanzinger S, Barion R, et al.: Patient and disease characteristics of adult patients with type 1 diabetes in Germany: an analysis of the DPV and DIVE databases. Ther Adv Endocrinol Metab 2019;10:1-15.

12. Svedbo Engström M, Leksell J, Johansson U-B, et al.: Health-related quality of life and glycaemic control among adults with type 1 and type 2 diabetes-a nationwide cross-sectional study. Health Qual Life Outcomes 2019;17:1-11.

13. Mcknight JA, Wild SH, Lamb MJE, et al.: Glycaemic control of type 1 diabetes in clinical practice early in the 21st century: an international comparison. Diabet Med 2015;32:1036-1050.

14. Aronson R, Orzech N, Ye C, et al.: Specialist-led diabetes registries and prevalence of poor glycemic control in type 2 diabetes: the Diabetes Registry Outcomes Project for A1C reduction (DROP A1C). Diabetes Care 2016;39:17111717.

15. Aronson R, Li A, Brown RE, et al.: Optimizing diabetes self-management using the novel Skills, Confidence, and Preparedness Index (SCPI). Diabetes Care 2019;42:18731878.

16. Aronson R, Orzech N, Ye C, et al.: Specialist-led diabetes registries and predictors of poor glycemic control in type 2 diabetes: insights into the functionally refractory patient from the LMC Diabetes Registry database. J Diabetes 2016;8:76-85.

17. Diabetes Canada Clinical Practice Guidelines Committee: Diabetes Canada 2018 Clinical Practice Guidelines for the Prevention and Management of Diabetes in Canada. Can J Diabetes 2018;42:S1-S325.

18. American Diabetes Association: Standards of Medical Care in Diabetes-2019. Diabetes Care 2019;42:S1-S2.

19. Willers C, Iderberg H, Axelsen M, et al.: Sociodemographic determinants and health outcome variation in individuals with type 1 diabetes mellitus: a register-based study. PLoS One 2018;13:1-13.

20. Miller KM, Beck RW, Foster NC, Maahs DM: HbA1c levels in type 1 diabetes from early childhood to older adults: a deeper dive into the influence of technology and socio-economic status on HbA1c in the T1D Exchange Clinic Registry Findings. Diabetes Technol Ther 2020; [Epub ahead of print]; doi:10.1089/dia.2019.0393.

21. Charalampopoulos D, Hermann JM, Svensson J, et al.: Exploring variation in glycemic control across and within eight high-income countries: a cross-sectional analysis of 64,666 children and adolescents with type 1 diabetes. Diabetes Care 2018;41:1180-1187. 
22. Fung $\mathrm{CH}$, Lim $\mathrm{Y}-\mathrm{W}$, Mattke $\mathrm{S}$, et al.: Systematic review: the evidence that publishing patient care performance data improves quality of care. Ann Intern Med 2008;148:111.

23. Benkhadra K, Alahdab F, Tamhane SU, et al.: Continuous subcutaneous insulin infusion versus multiple daily injections in individuals with type 1 diabetes: a systematic review and meta-analysis. Endocrine 2017;55:77-84.

24. Yeh H, Brown TT, Maruthur N, et al.: Comparative effectiveness and safety of methods of insulin delivery and glucose monitoring for diabetes mellitus. Ann Intern Med 2012;157:336-347.

25. Beck RW, Bergenstal RM, Cheng P, et al.: The relationships between time in range, hyperglycemia metrics, and HbA1c. J Diabetes Sci Technol 2019;13:614-626.

26. Vigersky RA, McMahon C: The relationship of hemoglobin A1C to time-in-range in patients with diabetes. Diabetes Technol Ther 2019;21:81-85.

27. Battelino T, Danne T, Bergenstal RM, et al.: Clinical targets for continuous glucose monitoring data interpretation: recommendations from the International Consensus on Time in Range. Diabetes Care 2019;42:1593-1603.

28. Šoupal J, Petruželková L, Flekač M, et al.: Comparison of different treatment modalities for type 1 diabetes, including sensor-augmented insulin regimens, in 52 weeks of followup: a COMISAIR Study. Diabetes Technol Ther 2016;18: 532-538.

29. Miller KM, Beck RW, Bergenstal RM, et al.: Evidence of a strong association between frequency of self-monitoring of blood glucose and hemoglobin A1c levels in T1D exchange clinic registry participants. Diabetes Care 2013;36:2009_ 2014.

30. Schwandt A, Best F, Biester T, et al.: Both the frequency of $\mathrm{HbA} 1 \mathrm{c}$ testing and the frequency of self-monitoring of blood glucose predict metabolic control: a multicentre analysis of 15199 adult type 1 diabetes patients from Germany and Austria. Diabetes Metab Res Rev 2017;33: e2908.

31. American Diabetes Association: Diabetes technology: standards of medical care in diabetes-2019. Diabetes Care 2019;42:S71-S80.

32. DiMeglio LA, Acerini CL, Codner E, et al.: ISPAD Clinical Practice Consensus Guidelines 2018: glycemic control targets and glucose monitoring for children, adolescents, and young adults with diabetes. Pediatr Diabetes 2018;19: $105-114$.

33. Ministry of Health and Long-term Care: Insulin Pump and Supplies Policy and Administration Manual. Ministry of Health \& Long-Term Care, Toronto, Ontario. 2016.

Address correspondence to: Ronnie Aronson, MD LMC Diabetes and Endocrinology 1929 Bayview Avenue

Toronto M4G $3 E 8$

Ontario

Canada

E-mail: ronnie.aronson@lmc.ca 